\title{
Site-Selective Synthesis of 3,17-Diaryl-1,3,5,16-estratetraenes
}

\author{
Stefan Joppa \\ Peter Ehlers ${ }^{\mathrm{a}, \mathrm{b}}$ \\ Eva Frank ${ }^{c}$ \\ Erzsébet Mernyák ${ }^{c}$ \\ Gyula Schneider \\ János Wölfling ${ }^{c}$ \\ Alexander Villinger ${ }^{\mathrm{a}}$ \\ Peter Langer*a,b (i)
}

a Universität Rostock, Institut für Chemie, Albert-Einstein-Str. 3a, 18059 Rostock, Germany

peter.langer@uni-rostock.de

b Leibniz-Institut für Katalyse e.V. an der Universität Rostock,

Albert-Einstein-Str. 29a, 18059 Rostock, Germany

' Department of Organic Chemistry, University of Szeged,

Dóm tér 8, 6720 Szeged, Hungary

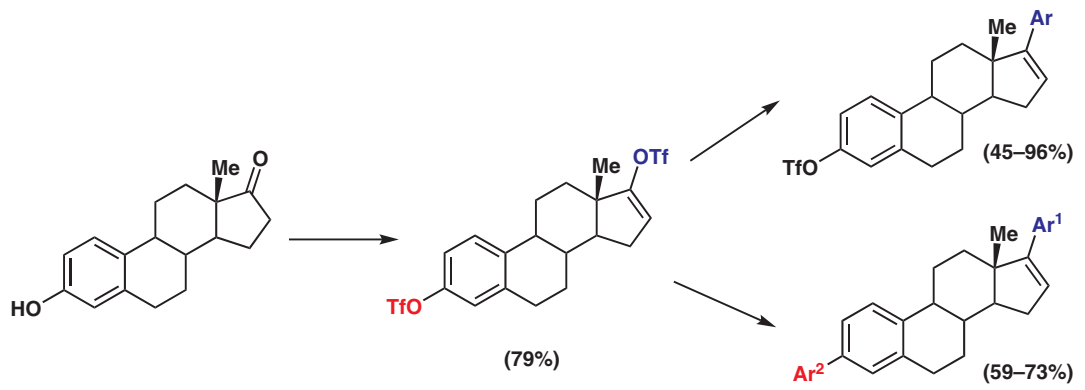

Received: 04.12.2018

Accepted: 09.01.2019

Published online: 07.02.2019

DOI: 10.1055/s-0037-1611720; Art ID: st-2018-d0786-

Abstract A straightforward, site-selective arylation of the bis(triflate) of estrone by Suzuki-Miyaura reactions has been developed. Monoarylation occurs selectively at the D-ring with good to excellent yield. Such products were exemplarily employed for the synthesis of estrones containing two different aryl substituents.

Key words palladium, site selectivity, steroids, Suzuki-Miyaura reaction

The functionalisation of natural products has been always a tool to develop novel medicinal drugs with improved activity, selectivity, stability, and/or better bioavailability. Such semisynthetic approaches are common in pharmaceutical research as they lower the number of synthetic steps and the need to introduce certain functionalities. For example, chiral centres are already incorporated and allow further derivatisation. In this regard, steroids have been intensively studied as privileged structures addressing various receptors with diverse biological functions (Figure 1). ${ }^{1}$

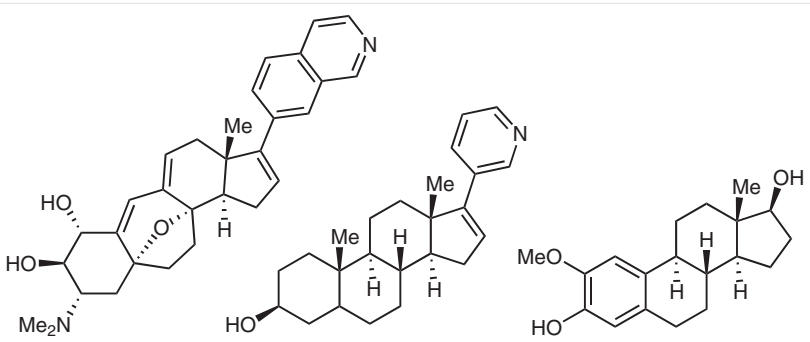

cortistatin A

abiraterone

2-methoxyestradiol

Figure 1 Naturally occurring steroids with anticancer activity
For instance, cortistatins are natural occurring steroids isolated from the marine sponge Corticium simplex and found great attention in anticancer research. Such compounds show potent antiangiogenic effects and inhibit proliferation of human umbilical vein endothelial cells in low nanomolar range. ${ }^{2}$ Hence, several derivatives have been synthesized and studied for their anticancer activity. ${ }^{1,3}$

Structurally related estrones, 1,3,5(10)-estratrien-3-ol17-one, as steroidal hormones, among other activities responsible for menstrual and estrous reproductive cycles of human females, have been functionalised on various positions leading to diverse pharmaceutical activity. In particular, ethynyl estradiol is commonly used as a component of oral contraceptive pills. ${ }^{4}$ In contrast, 3-alkynylestrones show antiviral activity and inhibition of alkaline phosphatases. ${ }^{5}$ Other studies revealed that functionalisation of positions two or three leads to antiangiogenic and antiproliferative activity and inhibition of steroid sulfatase. Undesired estrogenic effects were not observed and, hence, these molecules can be used in anticancer therapies. ${ }^{2 f, 6}$ Moreover, introduction of boronic pinacol esters at positions 3 and 17 lead to induction of gene expression in response to hydrogen peroxide and, therefore, enable selective detection of hydrogen peroxide in living mammalian cells. ${ }^{7}$

In recent years, we studied the functionalisation of steroids by palladium-catalysed cross-coupling reactions. ${ }^{5 \mathrm{~b}, 8}$ In 2010, Sun et al. reported the twofold Sonogashira reaction of the bis(triflate) of estradione. ${ }^{9}$ Due to the biological importance of 3- and 17-funtionalised 1,3,5,16-estratetraenes, we decided to study arylation reactions of the bis(triflate) of estrone, i.e., of 3,17-(trifluorosulfonyloxy)-1,3,5,16estratetraene. Herein, we report what are, to the best of our knowledge, the first Suzuki-Miyaura reactions of the bis(triflate) of estradione. The reactions proceed in good yields and with excellent site selectivity. The synthesis of 3or 17 -monoarylated $1,3,5,16$-estratetraenes by classical 
methods has been previously reported. ${ }^{6 f, 10,11}$ However, these reactions have, in most cases, a narrow product scope.

3,17-(Trifluorosulfonyloxy)-1,3,5,16-estratetraene (1) was synthesised from estrone in $79 \%$ yield in one-step according to a known method. ${ }^{12}$ Subsequently, we studied the Suzuki-Miyaura reaction of $\mathbf{1}$ with 3.0 equiv. of (4-methoxy)boronic acid using different reaction conditions. At the beginning, we employed 1,4-dioxane as the solvent and
SPhos as the ligand as these conditions were previously successfully employed for reactions of triflates of estrones (Table 1). ${ }^{8 b}$ However, the desired diarylated product 2a could be isolated in only $36 \%$ yield (Table 1 , entry 1 ). The low yield can be explained by the fact that a significant amount of monoarylated product 3a was formed (up to $30 \%)$. Therefore, in the following, we changed the solvent. The reaction in toluene gave product $\mathbf{2 a}$ in an acceptable yield of $68 \%$ yield (Table 1 , entry 2 ), while the reaction in

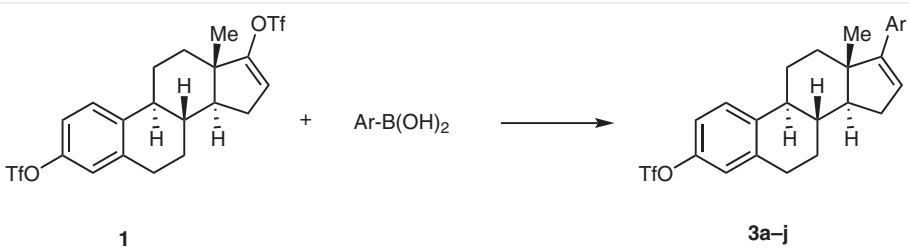

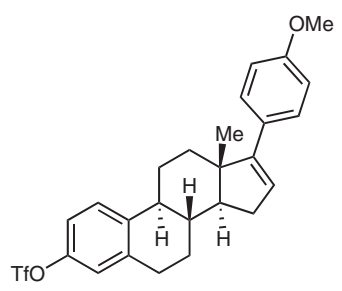

3a (96\%)

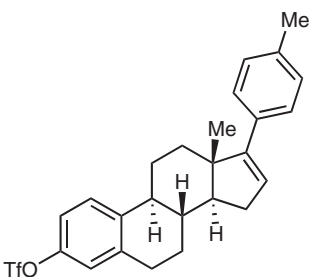

3b $(95 \%)$<smiles>Cc1cccc(C2=CCC3C4CCc5cc(O)ccc5C4CCC23N)c1</smiles>

$3 c(68 \%)$<smiles>COc1ccc2c(c1)CCC1C2CC[C@]1(N)c1cccc(O)c1</smiles>

$3 c(68 \%)$<smiles>COc1ccc(C2=CCC3C4CCc5cc(O)ccc5C4CC[C@]23N)cc1</smiles>

3 e $(80 \%)$<smiles>[M]C12CC[C@H]3c4ccc(O)cc4CC[C@]3(C)[C@@H]1CC=C2c1ccc(F)cc1</smiles>

$3 f(66 \%)$

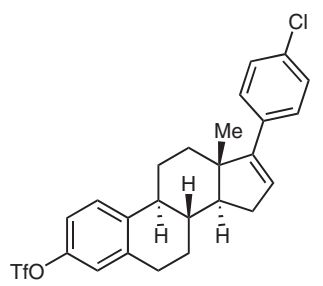

$3 g(45 \%)$<smiles>CCCC1(C)CCC2c3ccc(O)cc3CC[C@@H]2[C@H]1CC</smiles>

3j $(71 \%)$

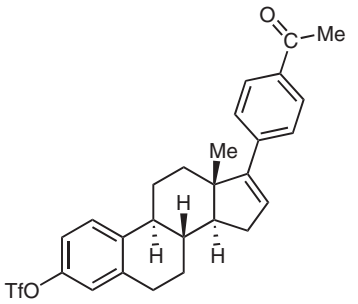

3h (55\%)<smiles>C=Cc1ccc(C2=CCC3C4CCc5cc(O)ccc5C4CC[C@]23N)cc1</smiles>

3i (86\%)

Scheme 1 Synthesis of $\mathbf{3 a - j}$. Isolated yields are given. 
Table 1 Optimisation - Synthesis of $\mathbf{2 a}$ and $\mathbf{3} \mathbf{a}^{\mathbf{a}}$

\begin{tabular}{|c|c|c|c|c|c|c|}
\hline & 1 & & $\mathrm{MeO}^{-}$ & & 3a & \\
\hline$\#$ & Ligand (mol\%) & $\begin{array}{l}\mathrm{ArB}(\mathrm{OH})_{2} \\
\text { (equiv.) }\end{array}$ & Solvent & Temp $\left({ }^{\circ} \mathrm{C}\right)$ & Yield 2a (\%) & Yield 3a (\%) \\
\hline 1 & SPhos (10) & 3.0 & 1,4-dioxane & 100 & 36 & 31 \\
\hline 2 & SPhos (10) & 3.0 & toluene & 100 & 68 & 23 \\
\hline 3 & SPhos (10) & 3.0 & xylene & 130 & 22 & 11 \\
\hline 4 & $n$ BuPAd $_{2}(10)$ & 3.0 & toluene & 100 & 0 & 96 \\
\hline 5 & $n \operatorname{BBPAd}_{2}(10)$ & 1.5 & toluene & 100 & 0 & 77 \\
\hline
\end{tabular}

${ }^{a}$ Conditions: $\mathrm{Pd}(\mathrm{OAc})_{2}(5 \mathrm{~mol} \%)$, ligand, $\mathrm{K}_{3} \mathrm{PO}_{4}$ solvent, $20 \mathrm{~h}$.

xylene at elevated temperature resulted in lower yields (Table 1, entry 3 ). Interestingly, a change of the ligand and use of CataXium A $\left(n \mathrm{BuPAd}_{2}\right)$, resulted in a complete change of the product distribution and formation of monoarylated product 3a in nearly quantitative yield (96\%, Table 1, entry 4). Product 3a was isolated in only 77\% yield when 1.5 instead of 3.0 equiv. of the boronic acid was employed. The structure of product 3a was confirmed by NOESY experiments showing a correlation between the anisol protons and the $13 \beta$-methyl group of the estrone moiety.
After optimisation of the conditions, we turned our attention to the impact of the functional groups attached to the arylboronic acid on the reaction outcome (Scheme 1). ${ }^{13}$

At the beginning, we studied the sterical influence of the substituents. The reaction of $\mathbf{1}$ with $p$-tolylboronic acid gave product $\mathbf{3 b}$ in excellent yield (95\%), while product $\mathbf{3 c}$, containing a meta-substituted methyl group, was isolated in only $68 \%$ yield. ${ }^{14}$ The use of ortho-tolylboronic acid was unsuccessful as no conversion was observed. This steric effect might be explained by the presence of the methyl group located in position 13 of the estrone core structure.<smiles>[M]C12CCC3c4ccc(O)cc4CCC3(C1)C1CC=C(c3ccc(F)cc3)C12</smiles>

$3 f$<smiles>[M]C12CCC3c4ccc(O)cc4CCC3(C1)C1CC=C(c3ccsc3)C12</smiles>

3j<smiles>COc1ccc(OC)cc1</smiles><smiles>COc1ccc(OC)cc1</smiles><smiles>COc1ccc(-c2ccc3c(c2)CC[C@@H]2C3CC[C@]3(N)C(c4ccc(F)cc4)=CC[C@H]23)cc1</smiles><smiles>COc1ccc(-c2ccc3c(c2)CCC2C3CCC3(C)C(c4ccsc4)=CCC23)cc1</smiles>

Scheme 2 Synthesis of 3,17-diaryl-1,3,5,17-estratetraenes 4a and 4b. Conditions: $\mathrm{Pd}(\mathrm{OAc})_{2}(5 \mathrm{~mol} \%), \mathrm{SPhos}(10 \mathrm{~mol} \%)$, toluene, $100{ }^{\circ} \mathrm{C}, 20 \mathrm{~h}$. 
The electronic effects were next studied. Electron-poor arylboronic acids gave generally lower yields, due to their lower reactivity in the transmetalation step. However, the developed reaction conditions are consistent with the employment of various functional groups, such as the acetyl $(\mathbf{3 h})$, chloro (3g), and vinyl groups (3i) as well as heterocyclic groups $(\mathbf{3 j})$.

Finally, we exemplarily studied the synthesis of 3,17diaryl-1,3,5,16-estratetraenes starting from $\mathbf{3} \mathbf{f}$ and $\mathbf{3 j}$. Using our optimised conditions for the diarylation (Table 1, entry 2 ), we synthesized compounds $\mathbf{4 a}$ and $\mathbf{4 b}$ in moderate to good yields (Scheme 2).

The structures of compounds $\mathbf{4 a}$ and $\mathbf{4 b}$ gave suitable single crystals for X-ray analysis which provided an independent proof that the first arylation takes place site-selectively at the five-membered ring of the steroidal framework (Figure 2). ${ }^{15}$

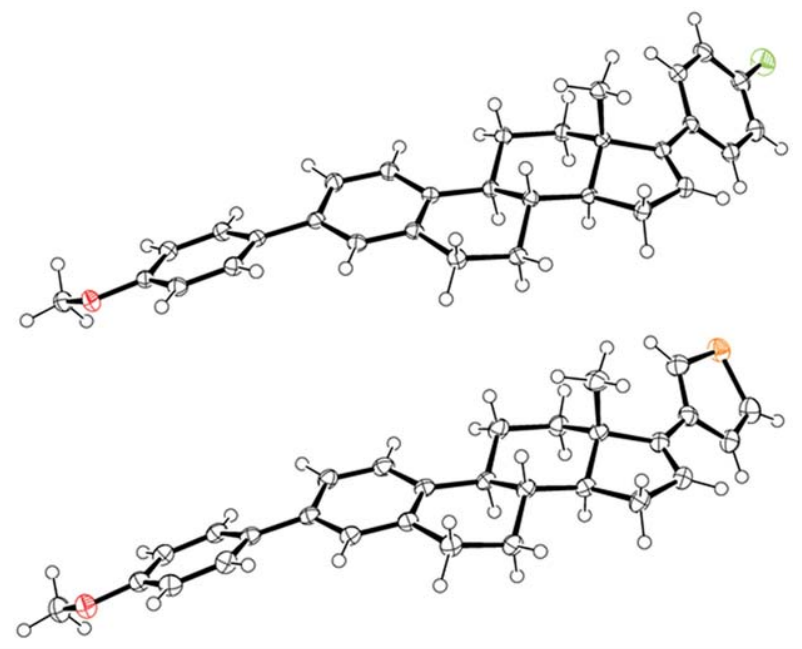

Figure 2 ORTEPs of compounds $\mathbf{4 a}$ (top) and $\mathbf{4 b}$ (bottom).

In conclusion, we developed an efficient methodology for the selective synthesis of arylated estratetraenes. The first arylation steps occurs selectively at position 17 and allows for further derivatisation at the second triflate group located at the A-ring.

\section{Funding Information}

Financial support by the BMBF (Response - Zwanzig20) is gratefully acknowledged.

\section{Supporting Information}

Supporting information for this article is available online at https://doi.org/10.1055/s-0037-1611720.

\section{References and Notes}

(1) Shi, J.; Shigehisa, H.; Guerrero, C. A.; Shenvi, R. A.; Li, C.-C.; Baran, P. S. Angew. Chem. Int. Ed. 2009, 48, 4328.

(2) (a) Aoki, S.; Watanabe, Y.; Sanagawa, M.; Setiawan, A.; Kotoku, N.; Kobayashi, M. J. Am. Chem. Soc. 2006, 128, 3148. (b) Watanabe, Y.; Aoki, S.; Tanabe, D.; Setiawan, A.; Kobayashi, M. Tetrahedron 2007, 4074. (c) Aoki, S.; Watanabe, Y.; Tanabe, D.; Setiawan, A.; Arai, M.; Kobayashi, M. Tetrahedron Lett. 2007, 48, 4485. (d) Aoki, S.; Watanabe, Y.; Tanabe, D.; Arai, M.; Suna, H.; Miyamoto, K.; Tsujibo, H.; Tsujikawa, K.; Yamamoto, H.; Kobayashi, M. Bioorg. Med. Chem. 2007, 15, 6758. (e) Sato, Y.; Kamiyama, H.; Usui, T.; Saito, T.; Osada, H.; Kuwahara, S.; Kiyota, H. Biosci. Biotechnol. Biochem. 2008, 72, 2992. (f) Solum, E. J.; Cheng, J.-J.; Sørvik, I. B.; Paulsen, R. E.; Vik, A.; Hansen, T. V. Eur. J. Med. Chem. 2014, 85, 391.

(3) (a) Nicolaou, K. C.; Sun, Y.-P.; Peng, X.-. S.; Polet, D.; Chen, D. Y.-K. Angew. Chem. Int. Ed. 2008, 47, 7310. (b) Lee, H. M.; Nieto-Oberhuber, C.; Shair, M. D. J. Am. Chem. Soc. 2008, 130, 16864.

(4) Inhoffen, H. H.; Holhlweg, W. Naturwissenschaften 1938, 26, 96.

(5) (a) Schang, L. M.; Vincent, A. V. US 20120135954, 2012. (b) Ivanov, A.; Boldt, S.; un Nisa, Z.; Shah, S. J. A.; Ehlers, P.; Villinger, A.; Schneider, G.; Wölfling, J.; Rahman, Q.; Iqbal, J.; Langer, P. RSC Adv. 2016, 6, 11118.

(6) (a) Möller, G.; Duluca, D.; Gege, C.; Rosinus, A.; Kowalik, D.; Peters, O.; Droescher, P.; Elger, W.; Adamski, J.; Killisch, A. Bioorg. Med. Chem. Lett. 2009, 19, 6740. (b) Nussbaumer, P.; Billich, A. Med. Res. Rev. 2004, 24, 539. (c) Geisler, J.; Lonning, P. E. Cancer Res. 2005, 11, 2809. (d) Woo, L. W. L.; Leblond, B.; Purohit, A.; Potter, B. V. L. Bioorg. Med. Chem. 2012, 20, 2506. (e) Suwandi, L. S.; Agoston, G. E.; Shah, J. H.; Hanson, A. D.; Zhan, X. H.; LaVallee, T. M.; Treston, A. M. Bioorg. Med. Chem. Lett. 2009, 19, 6459. (f) Solum, E. J.; Cheng, J.-J.; Sylte, I.; Vik, A.; Hansen, T. V. RSC Adv. 2015, 5, 32497.

(7) Govan, J. M.; McIver, A. L.; Riggsbee, C.; Deiters, A. Angew. Chem. Int. Ed. 2012, 51, 9066.

(8) (a) Jopp, S.; Liesegang, M.; Ehlers, P.; Frank, E.; Schneider, G.; Wölfling, J.; Langer, P. Tetrahedron Lett. 2018, 59, 26. (b) Jopp, S.; Wallaschkowski, T.; Ehlers, P.; Frank, E.; Schneider, G.; Wölfling, J.; Mernyak, E.; Villinger, A.; Langer, P. Tetrahedron 2018, 74, 2825. (c) Jopp, S.; Liesegang, M.; Ehlers, P.; Frank, E.; Schneider, G.; Wölfling, J.; Villinger, A.; Langer, P. Synlett 2017, 28, 2647. (d) Ivanov, A.; Ejaz, S. A.; Syed, J. A.; Shah, P.; Ehlers, A.; Villinger, E.; Frank, G.; Schneider, J.; Wölfling, J.; Iqbal, P.; Langer, P. Bioorg. Med. Chem. 2017, 25, 949. (e) Riebe, S.; Jopp, S.; Ehlers, P.; Frank, E.; Schneider, G.; Wölfling, J.; Villinger, A.; Langer, P. Tetrahedron Lett. 2017, 58, 2801.

(9) Sun, Q.; Jiang, C.; Xu, H.; Zhang, Z.; Liu, L.; Wang, C. Steroids 2010, 75, 936.

(10) For arylation at position 3, see: (a) Tran, H.; McCallum, T.; Morin, M.; Barriault, L. Org. Lett. 2016, 18, 4308. (b) Wang, X.-Y.; Song, H. X.; Wang, S.-M.; Yang, J.; Qin, H.-L.; Jiang, X. Tetrahedron 2016, 72, 7606. (c) Iranpoor, N.; Panahi, F.; Jamedi, F. J. Organometal. Chem. 2015, 781, 6. (d) Li, X.-J.; Zhang, J.-L.; Geng, Y.; Jin, Z. J. Org. Chem. 2013, 78, 5078. (e) Chen, H.; Huang, Z.; Hu, X.; Tang, G.; Xu, P.; Zhao, Y.; Cheng, C.-H. J. Org. Chem. 2011, 76, 2338. (f) Guan, B.-T.; Wang, Y.; Li, B.-J.; Yu, D.-G.; Shi, Z.-J.J. Am. Chem. Soc. 2008, 130, 14468. (g) Lipshutz, B. H.; Petersen, T. B.; Abela, A. R. Org. Lett. 2008, 10, 1333. (h) Ciattini, P. G.; Morera, E.; Ortar, G. Tetrahedron Lett. 1993, 33, 4815.

(11) For arylation at position 17, see: (a) Lei, C.; Yip, Y. J.; Zhou, J. S. J. Am. Chem. Soc. 2017, 139, 6086. (b) Li, J.; Knochel, P. Angew. Chem. Int. Ed. 2018, 57, 11436. (c) Hamze, A.; Brion, J.-D.; Alami, 
M. Org. Lett. 2012, 14, 2782. (d) Sun, C.-L.; Wang, Y.; Zhou, X.; Wu, Z.-H.; Li, B.-J.; Guan, B.-T.; Shi, Z.-J. Chem. Eur. J. 2010, 16, 5844.

(12) Holt, D. A.; Levy, M. A.; Ladd, D. L.; Oh, H.-J.; Erb, J. M.; Heaslip, J. I.; Brandt, M.; Metcalf, B. W. J. Med. Chem. 1990, 33, 937.

(13) Onefold Suzuki-Miyaura Reaction of 1 - General Procedure Arylboronic acid $(0.60 \mathrm{mmol}), \mathrm{K}_{3} \mathrm{PO}_{4}(0.60 \mathrm{mmol}, 127 \mathrm{mg})$, $\mathrm{Pd}(\mathrm{OAc})_{2}$ (5 mol\%, $2.2 \mathrm{mg}$ ), and cataCXium $A \circledast(10 \mathrm{~mol} \%, 7.2 \mathrm{mg})$ were weighed into a pressure tube under argon. Compound $\mathbf{1}$ $(0.20 \mathrm{mmol}, 107 \mathrm{mg})$ was dissolved in toluene $(4 \mathrm{~mL})$ and added to the pressure tube. The reaction was stirred at $100^{\circ} \mathrm{C}$ for $20 \mathrm{~h}$. After cooling to room temperature, the solution was diluted with water and extracted with ethyl acetate $(3 \times 10 \mathrm{~mL})$. The crude products $\mathbf{3 a}-\mathbf{j}$ were purified by column chromatography. 17-(4-Methylphenyl)-3-(trifluoromethylsulfonyloxy)-13及estra-1,3,5(10),16-tetraene (3b)

Compound $\mathbf{3 b}$ was synthesized according to the general procedure using 4-methylphenylboronic acid $(0.60 \mathrm{mmol}, 82 \mathrm{mg})$ and purified via column chromatography (heptane/dichloromethane, 10:1); yield $90 \mathrm{mg}(95 \%) ;[\alpha]_{D}{ }^{28}-24.9\left(\mathrm{CHCl}_{3}, \beta=1.5 \mathrm{mg}\right.$ $\left.\mathrm{mL}^{-1}\right)$. ${ }^{1} \mathrm{H}$ NMR $\left(300 \mathrm{MHz}, \mathrm{CDCl}_{3}\right): \delta=1.07\left(\mathrm{~s}, 3 \mathrm{H}^{-\mathrm{CH}_{3}}\right), 1.48-$ $1.59\left(\mathrm{~m}, 1 \mathrm{H}, \mathrm{CH}_{\text {Alkyl }}\right), 1.67-1.82\left(\mathrm{~m}, 4 \mathrm{H}, \mathrm{CH}_{\text {Alkyl }}\right), 1.99-2.26(\mathrm{~m}, 3$ $\left.\mathrm{H}, \mathrm{CH}_{\text {Alkyl }}\right), 2.29-2.40\left(\mathrm{~m}, 6 \mathrm{H}, \mathrm{CH}_{3}+\mathrm{CH}_{\text {Alkyl }}\right), 2.94-2.97(\mathrm{~m}, 2 \mathrm{H}$, $\left.\mathrm{CH}_{\text {Alkyl }}\right), 5.92\left(\mathrm{dd},{ }^{3} \mathrm{~J}=3.19 \mathrm{~Hz},{ }^{3} \mathrm{~J}=1.74 \mathrm{~Hz}, 1 \mathrm{H}, \mathrm{C}=\mathrm{CH}\right), 7.00-7.06$ $\left(\mathrm{m}, 2 \mathrm{H}, \mathrm{CH}_{\mathrm{Ar}}\right), 7.15\left(\mathrm{~d},{ }^{3} \mathrm{~J}=7.91 \mathrm{~Hz}, 2 \mathrm{H}, \mathrm{CH}_{\mathrm{Ar}}\right), 7.30-7.37(\mathrm{~m}, 3 \mathrm{H}$, $\left.\mathrm{CH}_{\mathrm{Ar}}\right) \cdot{ }^{13} \mathrm{C}$ NMR $\left(75 \mathrm{MHz}, \mathrm{CDCl}_{3}\right): \delta=16.6,21.1\left(\mathrm{CH}_{3}\right), 26.4,27.3$, 29.5, 31.2, 35.4 $\left(\mathrm{CH}_{2}\right), 36.8,44.2(\mathrm{CH}), 47.4(\mathrm{C}), 56.7(\mathrm{CH}), 118.1$ $(\mathrm{C}=\mathrm{CH}), 118.8\left(\mathrm{q},{ }^{1} \mathrm{~J}=320.8 \mathrm{~Hz}, \mathrm{CF}_{3}\right), 121.1,126.2,126.2,126.9$, $128.9\left(\mathrm{CH}_{\mathrm{Ar}}\right), 134.2,136.5,139.5,141.2,147.5\left(\mathrm{C}_{\mathrm{Ar}}\right), 154.7$ $(\mathrm{C}=\mathrm{CH}) .{ }^{19} \mathrm{~F}$ NMR $\left(282 \mathrm{MHz}, \mathrm{CDCl}_{3}\right): \delta=-72.97$. IR (ATR): $\mathrm{v}=$ $2930(\mathrm{w}), 2850(\mathrm{w}), 1488(\mathrm{w}), 1416(\mathrm{~s}), 1250(\mathrm{~m}), 1201(\mathrm{~s})$, $1139(\mathrm{~s}), 925(\mathrm{~s}), 886(\mathrm{~m}), 836(\mathrm{~m}), 797(\mathrm{~s}), 700(\mathrm{w}), 599(\mathrm{~s}), 511$ (w), $486(\mathrm{~m}) \mathrm{cm}^{-1}$. MS (EI, $\left.70 \mathrm{eV}\right): \mathrm{m} / z(\%)=477$ (16), $476(83)$ [M+], 461 (58), 291 (24), 170 (34), 169 (75), 165 (23), 157 (40), 155 (33), 153 (29), 141 (39), 131 (20), 130 (28), 129 (51), 128
(44), 116 (25), 115 (89), 105 (44), 91 (47), 77 (15), 69 (100) $\left[\mathrm{CF}_{3}{ }^{+}\right]$. HRMS (EI, $70 \mathrm{eV}$ ): $\mathrm{m} / \mathrm{z}$ calcd for $\mathrm{C}_{26} \mathrm{H}_{27} \mathrm{~F}_{3} \mathrm{O}_{3} \mathrm{~S}\left[\mathrm{M}^{+}\right]$: 476.16275; found: 476.16212 .

(14) Twofold Suzuki-Miyaura Reaction - General Procedure 4-Methoxyphenylboronic acid $(0.60 \mathrm{mmol}, 91 \mathrm{mg}), \mathrm{K}_{3} \mathrm{PO}_{4}(0.60$ $\mathrm{mmol}, 127 \mathrm{mg}$ ), $\mathrm{Pd}(\mathrm{OAc})_{2}$ (5 mol\%, $2.2 \mathrm{mg}$ ), and SPhos (10 mol\%, $8.2 \mathrm{mg}$ ) were weighed into a pressure tube under argon. Compound $3 \mathbf{f}(0.20 \mathrm{mmol}, 96 \mathrm{mg})$ was dissolved in toluene $(4 \mathrm{~mL})$ and added to the pressure tube. The reaction was stirred at $100{ }^{\circ} \mathrm{C}$ for $20 \mathrm{~h}$. After cooling to room temperature, the solution was diluted with water and extracted with ethyl acetate $(3 \times 10 \mathrm{~mL})$. The crude product $\mathbf{4 a}$ was purified via column chromatography (heptane/dichloromethane, 5:1); yield $64 \mathrm{mg}(73 \%) .[\alpha]_{D}{ }^{30}=28.5$ $\left(\mathrm{CHCl}_{3}, \beta=1.1 \mathrm{mg} \mathrm{mL}^{-1}\right)$; mp 181-183 ${ }^{\circ} \mathrm{C} .{ }^{1} \mathrm{H}$ NMR $(300 \mathrm{MHz}$, $\left.\mathrm{CDCl}_{3}\right): \delta=1.08\left(\mathrm{~s}, 3 \mathrm{H}, \mathrm{CH}_{3}\right), 1.52-1.60\left(\mathrm{~m}, 1 \mathrm{H}, \mathrm{CH}_{\text {Alkyl }}\right), 1.70-$ $1.90\left(\mathrm{~m}, 4 \mathrm{H}, \mathrm{CH}_{\text {Alkyl }}\right), 2.01-2.22\left(\mathrm{~m}, 3 \mathrm{H}, \mathrm{CH}_{\text {Alkyl }}\right), 2.32-2.39(\mathrm{~m}, 3$ $\left.\mathrm{H}, \mathrm{CH}_{\text {Alkyl }}\right), 3.00-3.06\left(\mathrm{~m}, 2 \mathrm{H}, \mathrm{CH}_{\text {Alkyl }}\right), 3.87\left(\mathrm{~s}, 3 \mathrm{H}, \mathrm{OCH}_{3}\right), 5.92$ $\left(\mathrm{dd},{ }^{3} \mathrm{~J}=3.15 \mathrm{~Hz},{ }^{3} \mathrm{~J}=1.73 \mathrm{~Hz}, 1 \mathrm{H}, \mathrm{C}=\mathrm{CH}\right), 6.98-7.06(\mathrm{~m}, 4 \mathrm{H}$, $\left.\mathrm{CH}_{\mathrm{Ar}}\right), 7.33-7.42\left(\mathrm{~m}, 5 \mathrm{H}, \mathrm{CH}_{\mathrm{Ar}}\right), 7.53-7.56\left(\mathrm{~m}, 2 \mathrm{H}, \mathrm{CH}_{\mathrm{Ar}}\right) \cdot{ }^{13} \mathrm{C}$ $\operatorname{NMR}\left(75 \mathrm{MHz}, \mathrm{CDCl}_{3}\right): \delta=16.6\left(\mathrm{CH}_{3}\right), 26.6,27.7,29.6,31.3,35.5$ $\left(\mathrm{CH}_{2}\right), 37.2,44.5(\mathrm{CH}), 47.6(\mathrm{C}), 55.3\left(\mathrm{OCH}_{3}\right), 56.9(\mathrm{CH}), 114.1$ $\left(\mathrm{CH}_{\mathrm{Ar}}\right), 115.0\left(\mathrm{~d},{ }^{2} \mathrm{~J}=21.1 \mathrm{~Hz}, \mathrm{CH}_{\mathrm{Ar}}\right), 124.0,125.5\left(\mathrm{CH}_{\mathrm{Ar}}\right), 127.0$ $(\mathrm{C}=\mathrm{CH}), 127.3,128.0\left(\mathrm{CH}_{\mathrm{Ar}}\right), 128.2\left(\mathrm{~d},{ }^{3} \mathrm{~J}=7.72 \mathrm{~Hz}, \mathrm{CH}_{\mathrm{Ar}}\right), 133.3$ $\left(\mathrm{d},{ }^{4} \mathrm{~J}=3.34 \mathrm{~Hz}, \mathrm{C}_{\mathrm{Ar}}\right), 133.7,137.0,138.2,139.0\left(\mathrm{C}_{\mathrm{Ar}}\right), 154.0$ $(\mathrm{C}=\mathrm{CH}), 158.9\left(\mathrm{C}_{\mathrm{Ar}}\right), 161.9\left(\mathrm{~d},{ }^{1} \mathrm{~J}=245.6 \mathrm{~Hz}, \mathrm{C}-\mathrm{F}\right) .{ }^{19} \mathrm{~F}$ NMR $\left(282 \mathrm{MHz}, \mathrm{CDCl}_{3}\right): \delta=-115.92$. IR (ATR): $v=2924(\mathrm{w}), 2904(\mathrm{w})$, $2850(\mathrm{w}), 1601(\mathrm{w}), 1508(\mathrm{~m}), 1490(\mathrm{~m}), 1279(\mathrm{w}), 1244(\mathrm{~m})$, $1222(\mathrm{~m}), 1179(\mathrm{~m}), 1038(\mathrm{~m}), 843(\mathrm{~m}), 807(\mathrm{~s}), 647(\mathrm{w}), 556$ (m), $521(\mathrm{w}), 504(\mathrm{w}) \mathrm{cm}^{-1}$. MS (EI, $\left.70 \mathrm{eV}\right): \mathrm{m} / z(\%)=439(28)$, 438 (100) [ $\left.\mathrm{M}^{+}\right], 250$ (15), 249 (21), 247 (15), 173 (21), 165 (12), 133 (13), 109 (18). HRMS (EI, $70 \mathrm{eV}$ ): $\mathrm{m} / z$ calcd for $\mathrm{C}_{31} \mathrm{H}_{31} \mathrm{FO}$ $\left[\mathrm{M}^{+}\right]$: 438.23535; found: 438.23428 .

(15) CCDCs 1882186 and 1882187 contain supplementary crystallographic data for this paper. These data can be obtained free of charge from The Cambridge Crystallographic Data Centre via www.ccdc.cam.ac.uk/getstructures. 